\title{
Le développement de compétences en conception de systèmes embarqués via l'apprentissage par problèmes et par projets
}

\author{
Philippe Mabilleau \\ Philippe.mabilleau@USherbrooke.ca \\ Département de Génie Électrique et de Génie informatique, Faculté de Génie, \\ Université de Sherbrooke, Sherbrooke, Québec, Canada, J1K 2R1
}

\begin{abstract}
RESUME Cet article montre comment le développement de compétences en conception de systèmes embarqués peut être réalisé dans le cadre d'une approche pédagogique par problèmes et par projets dans un programme d'ingénierie. Cette mise en œuvre a été effectuée au sein du programme de baccalauréat en génie informatique de l'Université de Sherbrooke qui s'offre entièrement selon cette approche pédagogique. Les systèmes embarqués constituent le thème d'une session d'étude au complet dans le programme. L'intégration des compétences liées aux systèmes embarqués se fait par le biais d'un projet impliquant le développement d'une application construite autour d'une unité mobile connectée à un serveur d'application via un lien sans fil et une passerelle IP. L'acquisition des compétences de base en systèmes embarqués se fait au travers d'une série de problématiques résolues par l'étudiant tout au long de la même session d'étude.
\end{abstract}

Mots clés : système embarqué, microcontrôleur, noyau temps réel, pile de protocole, lien sans fil, mise en réseau des systèmes embarqués, intégration de logiciel, intégration logiciel matériel, apprentissage par problèmes et par projets, projet d'intégration, compétences en conception de projet.

\section{INTRODUCTION}

Dans le cadre d'une refonte complète de ses programmes de baccalauréat en génie électrique et en génie informatique la faculté de génie de l'Université de Sherbrooke a opté pour une utilisation «mur à mur $»^{1}$ de l'approche pédagogique par problèmes et par projets (APPI). Cette approche, inspirée du Problem Base Learning [1], est appliquée sur l'ensemble des deux programmes de $1^{\mathrm{er}}$ cycle du département de génie électrique et de génie informatique depuis septembre 2001 [2]. Cette réforme a débuté par une redéfinition des deux programmes à partir des compétences attendues des ingénieurs que l'on souhaitait former. Parmi ces compétences on va retrouver des compétences scientifiques et techniques propres aux systèmes embarqués ainsi que des compétences en conception applicables au cas du développement de ces mêmes systèmes.

Outre la redéfinition des finalités attendues pour les programmes réformés, l'introduction de l'approche par problèmes et par projets a débouché sur une mise en œuvre totalement nouvelle de la structure et du déroulement des activités au sein des programmes [2] [3]. L'organisation en session d'études de 4 mois qui existait déjà dans nos programmes, a été renforcée par la cohérence en structure uniformisée pour toutes les sessions et par la définition d'un thème moteur des apprentissages pour chaque session. Dans le programme de génie informatique une session a donc été consacrée aux systèmes embarqués qui, sans être absents des au-

1 Par cette formulation nous entendons sur l'ensemble des programmes ce qui implique qu'il n'y a plus aucune leçon magistrale. tres sessions, y seront vus en profondeur. En particulier les implications sur le développement et la conception de la présence de ressources restreintes sur ces systèmes, ainsi que la dépendance plus forte que l'on y retrouve entre le matériel et le logiciel, sont des éléments qui seront mis de l'avant.

Chaque session comporte un certain nombre, généralement 6 ou 7, de problématiques, appelées " unité d'APP », et que l'étudiant doit résoudre pour développement l'ensemble des compétences scientifiques et techniques liées au thème de la session. Le déroulement de la résolution de chaque problématique répond à une structure bien définie [3]. Un exemple de problématique proposée aux étudiants dans une unité d'APP est présenté dans [4].

La session comporte également un projet intégrateur permettant à l'étudiant d'exercer les compétences développées à l'occasion des problématiques dans un contexte différent soit celui de la réalisation complète d'un système. Donc en principe ce projet n'est pas le lieu d'acquisition de nouvelles compétences scientifiques et techniques, il est seulement l'occasion de les appliquer dans un nouveau contexte, et par la même indirectement il permet d'en parfaire le développement. Ce projet intégrateur a également comme objectif spécifique le développement de compétences propres à la gestion et au suivi de projets. De plus toutes les activités, soit les unités d'APP et le projet, sont également prétextes au développement de compétences intra personnelles et interpersonnelles comme la communication, le travail en équipe et l'analyse de ses besoins de formation et de ses stratégies d'apprentissage.

Dans cet article nous allons présenter les spécificités de mise en œuvre de l'approche par problèmes et par projets pour la session dédiée aux systèmes embarqués du programme de génie informatique. Après une présentation des compétences liées à 
l'informatique embarquée dont on vise le développement chez les étudiants durant la session, les unités d'APP et le projet sont présentés. En particulier la plate-forme technologique utilisée pour supporter les apprentissages ainsi que la finalité de la réalisation demandée aux étudiants sont présentées.

\section{LES COMPETENCES LIEES A L'INFORMATQIUE EMBARQUEE}

Un noyau de compétences liées à l'informatique embarquée a été identifié et intégré au corpus de compétences de l'ingénieur en génie informatique. La liste de ces éléments de compétence est donnée dans le tableau 1 . On y retrouve des compétences au niveau de la programmation et de l'exploitation de cartes à microcontrôleur, de la conception d'applications exploitant les fonctionnalités d'un noyau temps réel et de la programmation concurrente, de l'exploitation de dispositifs d'entrées/sorties sur un microcontrôleur et de l'intégration d'un système à microcontrôleur à un réseau informatique.

Exploiter une carte comportant un microcontrôleur et des circuits d'entrée/sortie en utilisant la documentation technique des composants et des logiciels qui la constituent

Développer et mettre au point un logiciel sur un système embarqué en utilisant des outils de développement croisés

Exploiter les sous-systèmes périphériques d'un microcontrôleur pour interfacer différents capteurs et actionneurs

Développer, mettre en œuvre et tester une application intégrant un noyau temps réel sur une plate-forme à microcontrôleur

Concevoir un système embarqué exploitant la programmation concurrente dans une application en temps réel

Concevoir, développer et mettre au point une application sur un système embarqué exploitant la connectivité à un réseau informatique

Exercer des capacités d'analyse, d'abstraction, de synthèse et de créativité en rapport avec la conception, le développement et la réalisation d'une application répartie mettant en œuvre une fonctionnalité distribuée sur un réseau informatique comportant au moins un système embarqué

Tableau 1: Les éléments de compétence en informatique embarquée

Plusieurs de ces compétences sont des variantes de compétences informatiques plus générales appliquées dans le contexte plus spécifique des systèmes embarqués. Même si l'apport conceptuel de ces compétences par rapport à la même compétence plus générale n'apparaît pas aux étudiants dans un premier temps, leur maîtrise fait appel à des méthodes nouvelles et très spécifiques et les choix et décisions qui y sont souvent associés se basent sur des métriques elles aussi propres aux systèmes embarqués. Par exemple la programmation en $\mathrm{C} / \mathrm{C}++$ des systèmes embarqués est très similaire quant au langage à la programmation sur des systèmes conventionnels, mais les outils de développement utilisés, en particulier via le développement croisé, et de mise au point sont différents. Un autre exemple est celui de la programmation concurrente et des mécanismes de synchronisation, déjà vus dans une autre session dans le contexte des systèmes d'exploitation, qui est révisé dans la session sur les systèmes embarqués via l'utilisation d'un noyau temps réel où les mécanismes de partage de l'unité centrale et de synchronisation sont beaucoup plus proches du code de l'application.

Souvent les compétences techniques développées à propos des systèmes embarqués permettent d'approfondir et de mieux assimiler les compétences plus générales en informatique. La transparence et les nombreuses fonctionnalités cachées des systèmes informatiques conventionnels n'offre pas un cadre didactique adéquat pour une assimilation de la mécanique interne de ceux-ci, alors que l'aspect plus élémentaire que l'on retrouve dans les systèmes embarqués est plus propice aux apprentissages à ce niveau. Ceci s'est vérifié par exemple avec les compétences en réseautique appliquées aux systèmes embarqués. L'exploitation des réseaux, via les interfaces de connexion (Socket), a été vue dans une session antérieure mais leur utilisation sur un système embarqué force les étudiants à mieux assimiler le fonctionnement interne de la pile de protocole TCP/IP. En particulier le rôle des modules ARP, DNS et DHCP a pu être décortiqué dans le mécanisme interne de fonctionnement de la pile alors que sur un ordinateur conventionnel ils apparaissent uniquement via leur interface de configuration si nécessaire.

\section{LES UNITES D'APP}

La session dont le thème est les systèmes embarqués comporte 6 unités d'APP dont 4 sont directement liées au développement de compétences propres à l'informatique embarquée.

Une première unité se veut une introduction au développement sur une carte à microprocesseur. Les compétences visées sont d'être capable d'exploiter une carte à microprocesseur et d'être capable de développer une application pour cette carte. Les concepts de développement croisé et de couche d'adaptation au matériel y sont mis de l'avant. La problématique consiste en la réalisation d'une application d'écoute de fichiers audio présents sur une carte compact flash (style lecteur MP3). Aucun noyau temps réel ni système d'exploitation ne sont utilisés, seule une bibliothèque d'accès à la carte compact flash formatée en format FAT16 est fournie. Une bibliothèque d'exécution standard (NEWLIB) est également fournie mais doit être adaptée et configurée pour la plate forme matérielle qui est une carte Zoom LH79520 de LogicPD. L'application est développée en $\mathrm{C}$, seul un code d'amorçage écrit en assembleur doit être vérifié et validé. En plus de se familiariser avec le processus de développement croisé et de mise au point sur la carte cible en utilisant un JTAG, les étudiants doivent assimiler le système de DMA du microcontrôleur pour exploiter le DAC audio utilisé pour l'écoute. Leur source d'information étant principalement la documentation technique de la carte et des circuits ils doivent 
développer des compétences au niveau de la lecture et de l'utilisation efficace de ce type de document.

Une seconde unité d'APP est consacrée à l'exploitation d'un noyau temps réel. La problématique est une simulation de la fonction de relais d'un satellite orbitant autour d'une planète (typiquement mars) et qui dispose seulement de quelques fenêtres temporelles de visibilité avec la terre et avec un robot au sol dont il doit relayer les données. Le satellite doit emmagasiner les données issues du robot au sol dans une mémoire tampon, et les retransmettre plus tard vers la terre lorsque celle-ci sera visible. Les deux opérations pouvant s'effectuer simultanément, la programmation concurrente avec mécanisme de synchronisation et d'échange de données entre tâches est requise. La simulation est réalisée avec un facteur d'échelle temporel pour permettre une exécution plus rapide que le temps réel avec des données réalistes. La même problématique est reprise plus tard dans la session pour appliquer des compétences en statistiques et évaluer la fiabilité du système. Le noyau temps réel utilisé est $\mu \mathrm{C} / \mathrm{OS}-\mathrm{II}$ développé par Micri $\mu \mathrm{m}$ et dont le code source est disponible dans un volume de référence [5]. La plate-forme utilisée est la même que celle utilisée dans l'APP précédente.

La troisième unité d'APP en relation avec les systèmes embarqués réalise un couplage entre les compétences sur les propriétés des matériaux et la réalisation de capteurs. La problématique consiste à mesurer le niveau d'un liquide dans un réservoir en utilisant un flotteur magnétique et une sonde à effet Hall. La même carte à microcontrôleur est utilisée pour faire l'acquisition et le traitement de la mesure.

Enfin une quatrième APP est directement liée avec l'intégration et l'exploitation d'une carte embarquée dans un réseau informatique. La problématique est celle de l'acquisition de données issues de capteurs dans un appartement intelligent, et de la transmission de celles-ci via un serveur distant exécutant une application collectant ces données. La configuration des paramètres d'acquisition (rythme, adresse du serveur, numéro du port) doit se faire via une interface WEB présente sur la carte. Là encore c'est la même carte qui est utilisée avec le noyau $\mu \mathrm{C} / \mathrm{OS}$-II et la pile associée $\mu \mathrm{C} / \mathrm{TCP}-\mathrm{IP}$. La pile est installée sur la carte mais n'est pas totalement configurée pour fonctionner sur les réseaux de l'Université. L'interface entre l'application, écrite en $\mathrm{C}$, et le serveur http de la pile $\mu \mathrm{C} / \mathrm{TCP}$-IP est à développer selon les spécifications fournies par $\mathrm{Mi}$ cri $\mu \mathrm{m}$. L'application de collection des données écrite en Java ainsi que le protocole à utiliser sont donnés.

La session est complétée par une problématique de mathématique sur les statistiques et une autre unité appliquant les statistiques à la fiabilité des systèmes temps réel.

\section{LE PROJET}

Le projet est non seulement le lieu d'intégration des compétences scientifiques et techniques acquises dans les unités d'APP, mais il est également l'occasion de parfaire des compétences transversales en développement et gestion de projet. Ces compétences visent de bonnes pratiques au niveau de la spécification, du développement et de la réalisation d'un système, notamment en ce qui concerne la production, l'exploitation et la diffusion d'une documentation standardisée autant pour le processus de développement du projet que pour le système développé lui-même. On y retrouve tout le savoir-faire au niveau de la planification et du suivi d'un projet. Les méthodologies utilisées sont inspirées de la pratique en génie du logiciel [6]. La figure 1 présente les champs de compétences développés dans le projet. Ils se divisent en trois axes : les compétences de réalisation du projet en tant que tel, les compétences de gestion du projet et les compétences de travail en équipe. Une trame temporelle de réalisation est donnée aux étudiants ; ceci délimite l'effort attribué au projet et permet une indentification et une planification des livrables associés à l'évaluation.

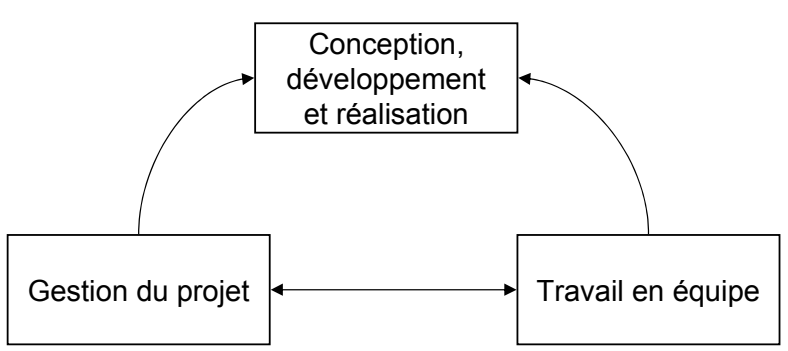

Figure 1: Les champs de compétences développés dans le projet

\subsection{La plate-forme utilisée}

Afin d'assurer la disponibilité des ressources matérielles et techniques requises pour le projet, le cadre technique de réalisation est imposé. Ceci nous assure que ce sont des composants déjà disponibles et pour lesquels nous avons l'expertise de soutien qui seront utilisés. De plus ce passage obligé par certains dispositifs techniques provoque une recontextualisation forcée des compétences développées dans les unités d'APP. L'architecture du système qui est ainsi imposé aux étudiants est présentée à la figure 2. Elle comporte :

- une unité mobile (PDA) comportant une interface utilisateur. Cette unité mobile peut recevoir différents dispositifs périphériques standards ou personnalisés ; - un lien sans fil entre cette unité mobile et une station de base ;

- une station de base servant de passerelle avec le réseau IP ;

- un serveur d'application. 


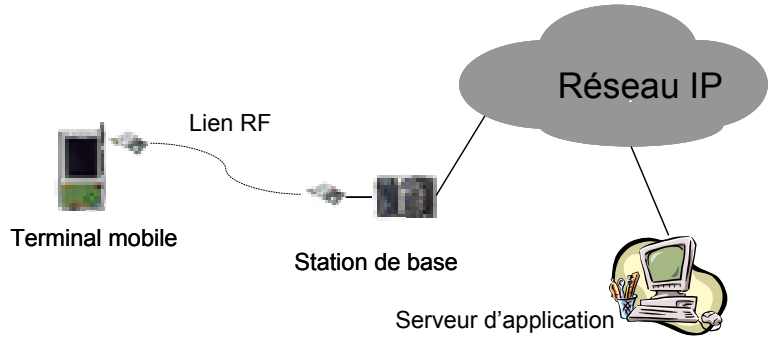

Figure 2 : L'architecture du système réalisé dans le projet

L'unité mobile est un assistant personnel ouvert fabriqué par Intrinsyc le CerfPDA. Ce modèle peut supporter Linux ou WindowsCE mais est utilisé dans le projet avec le noyau temps réel $\mu \mathrm{C} / \mathrm{OS}-\mathrm{II}$ de Micrijm afin de forcer un développement plus proche du matériel. Il dispose d'un écran tactile couleur de 3,8 pouces, d'un clavier alphanumérique, de facilités d'entrée/sortie sonore, d'une fente PCMCIA et de divers ports de communications (série, Bluetooth, IrDA, USB). Notre choix s'est arrêté vers ce type d'équipement en raison de son caractère ouvert et très versatile au niveau des interfaces, par opposition à un PDA conventionnel beaucoup moins accessible. Un certain nombre de dispositifs périphériques pour le PDA sont mis à la disposition des étudiants : récepteurs GPS, boussoles électronique, lecteurs de cartes magnétiques, lecteurs de code à barres... Il est également possible pour les étudiants de développer de petits dispositifs plus spécifiques et de les interfacer au PDA généralement via un port série. Par exemple une équipe a déjà interfacé des accéléromètres au PDA dans une application en relation avec la course automobile.

Le PDA est relié au réseau terrestre IP via un lien sans fil bâti autour de modules de communication RF. Les modules choisis intègrent toute la chaîne de communication de façon à offrir une connexion série entre un port du PDA et un port de la carte utilisée comme station de base. Il est important de remarquer que, de part la nature même du lien sans fil, la fiabilité de la connexion ne peut être garantie et c'est donc aux étudiants de développer une couche de protocole de type liaison de donnée, généralement inspirée de AHDLC, pour faire face aux aléas de la communication. Différents modules sont proposés offrant des puissances et des débits de transmission variés et permettant ainsi aux étudiants de faire un choix en fonction de la spécification des besoins.

La station de base est une carte Zoom LH79520 de LogicPD exploitée avec $\mu \mathrm{C} / \mathrm{OS}$-II et $\mu \mathrm{C} / \mathrm{TCP}-\mathrm{IP}$. Elle assure la connectivité au réseau IP jouant le rôle d'une passerelle pour les protocoles de niveau application entre le lien sans fil propriétaire et TCP ou UDP.

Enfin un serveur d'application hébergé sur une station de travail va compléter le système. L'emphase n'est pas mise sur le développement de ce serveur car celui-ci n'est pas du ressort des systèmes embarqués, thème de la session. La plupart des étudiants mettent en œuvre une base de données sur ce serveur pour entreposer les informations de l'application.

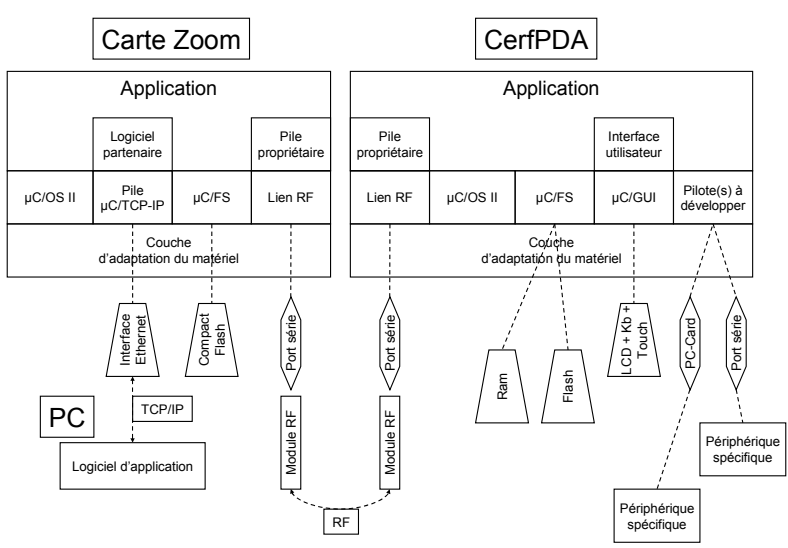

Figure 3 : L'architecture logicielle du système

Au niveau du logiciel l'architecture est également imposée. Celle-ci est présentée à la figure 3. Les logiciels de Micri $\mu \mathrm{m}, \mu \mathrm{C} / \mathrm{OS}-\mathrm{II}, \mu \mathrm{C} / \mathrm{TCP}-\mathrm{IP}, \mu \mathrm{C} / \mathrm{GUI}$ et $\mu \mathrm{C} / \mathrm{FS}$ sont utilisés en raison de l'accès que nous pouvons offrir autant au code source qu'au support technique. Ce choix évite également aux étudiants de s'égarer vers des solutions hasardeuses et pour lesquelles un support adéquat ne pourrait être offert. L'utilisation de solutions «clé en main » basée sur des systèmes d'exploitation et des ensembles logiciels de haut niveau, même si opérationnelles sur les platesformes matérielles utilisées, est interdite sauf pour le serveur d'application. Il est important que les étudiants aient une vision à relativement bas niveau de tout le système, c'est pourquoi on évite toute encapsulation de haut niveau même si elle répondrait mieux à des objectifs de productivité et de maintenance.

\subsection{La finalité de la réalisation}

Si l'architecture du système est imposée aux étudiants, sa finalité par contre reste leur choix. C'est chaque équipe d'étudiants qui doit déterminer quelle application le système développé doit mettre en oeuvre. Les motivations de ce choix sont basées sur des considérations sociales ou commerciales et il doit être supporté par un modèle d'affaire. Même si la viabilité économique ou sociale du projet n'est pas directement évaluée celui-ci doit offrir un minimum de réalisme et ne pas aller contre des considérations éthiques élémentaires. Le tableau 2 donne une liste d'applications réalisées lors des projets passés.

Les étudiants sont encouragés à identifier un client réel pour leur application et éventuellement à valider une étude des besoins par des entrevues avec des utilisateurs potentiels. Par exemple une équipe ayant décidé de développer un système d'aide pour effectuer les battues dans le cadre de la recherche en forêt de personnes disparues, a contacté les services de police et de la sécurité civile pour établir les besoins des utilisateurs et le cahier des charges du projet. 
Assistant pour la visite d'un musée

Stationnement intelligent sur le campus

Panier intelligent pour un supermarché

Aide pour la recherche de personnes disparues

Zoo guide

Assistant pour pilote de course automobile

Jeu de piste

Assistant pour ambulancier

Visite guidée du campus

Aide pour le personnel du service de l'équipement

Carte de golf électronique

Lecteur MP3 mobile

Assistant pour gardien de sécurité

\section{Tableau 2 : Exemples d'applications pour le projet}

Le choix de l'application de leur projet est une source de motivation importante pour les étudiants. L'intervention des professeurs se limite à quelques suggestions pour amorcer la créativité des étudiants et permet également de valider sommairement la faisabilité de ce qu'ils proposent. Les étudiants sont invité ensuite à rédiger un sommaire exécutif décrivant leur projet; la mise en situation de cet exercice serait celle d'une présentation devant des investisseurs potentiels d'où des consignes de concision, de clarté et de vocabulaire précis et accessible à tous.

\subsection{Le déroulement du projet}

Les équipes de projet sont d'une façon optimale composées de 8 étudiants ou étudiantes. Elles sont constituées sur une base volontaire par négociation entre les étudiants. Généralement le choix de l'application est un facteur de regroupement pour former les équipes.

Le temps consacré au projet est d'une journée par semaine pendant les 4 mois que dure la session d'étude. Des rencontres avec présentation sommaire des éléments clés et revue de projet sont organisées sur une base régulière avec le professeur. Un cadre de planification est proposé aux étudiants en particulier identifiant les livrables : sommaire exécutif, rapport intérimaire avec les spécifications à mi-session, rapport final et présentations de fin de session. Hors mis ces balises les étudiants ont carte blanche pour la planification détaillée et la gestion du processus.

L'évaluation est réalisée au travers des différents livrables et à l'aide d'un examen. Les livrables sont essentiellement des rapports écrits et une présentation orale à la fin du projet. Ce sont donc tous des éléments où c'est l'équipe qui est évaluée. L'évaluation individuelle se fait par le biais d'un examen portant sur les compétences de gestion et de planification d'une réalisation dans le contexte des systèmes embarqués. Même si ce sont des éléments de gestion de projet qui sont évalués en bout de ligne on dénote, dans cet examen, un couplage très fort avec des compétences techniques liées aux systèmes embarqués. Une présentation publique des projets est également organisée. Elle se déroule sous forme d'un salon où les étudiants ont l'occasion de présenter leurs réalisations à la commu- nauté universitaire. Les compétences liées au travail en équipe sont évaluées par les pairs dans chacune des équipes. Ce processus offre aux étudiants un mécanisme d'autorégulation pour éviter les dissonances au sein de chaque équipe relativement à la contribution des membres.

\section{CONCLUSION}

Le niveau de satisfaction et de réussite des étudiants est très élevé en rapport avec les contenus relatifs aux systèmes embarqués. Ils sont très motivés par le projet et y consacrent généralement beaucoup de temps et d'effort. Indirectement cette motivation se propage aux unités d'APP et se traduit par un intérêt accru pour les compétences techniques directement liées aux systèmes embarqués. Les conditions de déroulement du projet ainsi que leur motivation à obtenir un résultat fonctionnel, les font prendre conscience de la nécessité d'un processus de gestion rigoureux. Ceci est un élément facilitateur pour stimuler l'acquisition des compétences en gestion de projet.

D'une manière générale les compétences développées par les étudiants exposés à cette approche pédagogique sont remarquées par les employeurs, tant pendant les stages effectués au cours de leur scolarité que sur la marché du travail pour les deux promotions qui ont déjà complété la formation. Les étudiants se sont démarqués par leur autonomie et leur capacité à aborder les problèmes réels d'une façon plus efficace. Leurs aptitudes relatives à une meilleure exploitation des ressources disponibles (expertise humaine, documentation, temps etc...) ont été clairement identifiées. Dans un contexte relatif aux systèmes embarqués qui se caractérise par un éventail très large des expertises requises, allant du circuit électronique aux systèmes logiciels complexes, et par l'abondance des informations techniques ces aptitudes constituent un atout majeur.

Au niveau des ressources requises il faut distinguer les coûts relatifs au développement initial de la session des coûts récurrents associés à la prestation des différentes activités qui la constituent. Pour ce qui est des coûts associés à la mise en place de cette approche pour la session sur les systèmes embarqués, ils ont été comparables à ceux encourus pour le développement des autres sessions dans le programme lorsque le passage à l'APPI a été instauré. Il est intéressant de constater que la diminution des coûts des cartes à microcontrôleur et l'utilisation de logiciels libres permettent d'offrir aux étudiants des environnements de développement en nombre suffisant et facilement accessibles. L'effort en termes de ressources professorales demandé pour la prestation des activités est équivalent à ce qu'il était avec une méthode pédagogique traditionnelle. Seuls la nature des activités et leur agencement dans l'horaire sont différents. Ce constat est également valable pour l'ensemble des sessions de nos programmes en APPI. 
L'expérience du département de génie électrique et de génie informatique de l'Université de Sherbrooke montre qu'une approche pédagogique par problèmes et par projets (APPI) est très pertinente et bien appropriée pour le développement de compétences dans le domaine des systèmes embarqués.

\section{Bibliographie}

[1] D. Woods. Problem-Based Learning: resources to gain the most from PBL, Waterdown, ON, Canada, ISBN 0-9698725-2-6, revised 1996.

[2] Université de Sherbrooke. Description des programmes de génie informatique et de genie électrique. 2006.

http://www.usherbrooke.ca/Programmes/bacc /gelectri.html;

http://www.usherbrooke.ca/Programmes/bacc /ginform.html

[3] Noël Boutin et al, L'apprentissage par problèmes et par projets pour le développement des compétences professionnelles de l'ingénieur : l'exemple des programmes de génie électrique et de génie informatique de l'Université de Sherbrooke, soumis au $6^{\text {ième }}$ Colloque sur 1'Enseignement des Technologies et des Sciences de l'Information et des Systèmes CETSIS, 2007.

[4] Ahmed Khoumsi et Ruben Gonzalez-Rubio, Applying a Competency-and Problem-Based Approach for Learning Compiler Design, Journal of STEM Education, Volume 7 Issue 1 \& 2 January-June 2006, p. 24-33.

[5] Jean J. Labrosse, MicroC/OS-II The RealTime Kernel, Secomd Edition, CMP Books, 2002.

[6] Eric J. Braude, Software Engineering: An Object-Oriented Perspective, Willey, 2000. 